Asian Spine Journal

Vol. 1, No. 2, pp 75 79, 2007

\title{
Diagnosis and Treatment of Tuberclous Spondylitis and Pyogenic Spondylitis in Atypical Cases
}

\author{
Jae Sung Ahn, June Kyu Lee \\ Department of Orthopedic Surgery, School of Medicine, \\ Chungnam National University, Daejeon, Korea
}

\begin{abstract}
Study Design: A retrospective study.
Purpose: This is a study of the diagnosis and treatment of tuberculous spondylitis and pyogenic spondylitis in atypical cases. Overview of Literature: There have been several reports about clinical, hematological, pathological and radiological findings to differentiate pyogenic $\&$ tuberculous spondylitis.

Methods: We screened 55 patients diagnosed with tuberculous spondylitis and pyogenic spondylitis from January 1999 to June 2003. There were seven cases where it was difficult to make an accurate diagnosis. We reviewed the clinical manifestation, laboratory tests, radiological findings and confirmed the diagnoses by the use of biopsies and/or clinical response to treatment. Results: Four cases, which were initially diagnosed as pyogenic spondylitis, had a clinical presentation of fever $\left(37.4 \sim 38.5^{\circ} \mathrm{C}\right)$ on the day of hospitalization. These cases later turned out to be tuberculous spondylitis, as confirmed by an open biopsy and pathologic study. Three cases initially diagnosed as pyogenic spondylitis were treated with broad-spectrum antibiotics. Symptoms were aggravated in these cases, but improved after the use of an anti-tubercular drug. Bony union was observed in all cases in an averageof 4 months (range, 3 6 months).

Conclusions: In infectious spondylitis, it is important to establish an accurate diagnosis. An accurate diagnosis can be made by laboratory findings and by estimation of the response to treatment during follow-up. If there is no response or aggravation of symptoms despite treatment based on an initial diagnosis, the etiologic organism must be re-evaluated. A biopsy and observation of clinical response are needed to confirm the diagnosis.
\end{abstract}

Key Words: Tuberclous spondylitis, Pyogenic spondylitis

\section{Introduction}

The treatment of infectious spinal disease has recently progressed, but a delay in diagnosis (an average of 3 months), long duration of treatment (an average of 12 months) and financial concerns are the major problems in treatment ${ }^{1}$. Pyogenic spondylitis and tuberculous spondylitis are rare diseases, but they are major etiologies of infectious spondylitis, which can cause severe complications such as an irreversible neurological deficit and deformity in spinal alignment. Thus, accurate diagnosis in its early stage and proper antibiotic treatment based on identification of the infectious organism is required.

Though several studies have reported on the differential diagnosis between the two types of spondylitis based on clinical manifestation ${ }^{1-3}$, hematological tests, pathological tests and radiological tests, correct differentiation between the two diseases is still very difficult. We estimated the validity of differential diagnosis in seven selected cases: four cases that were initially diagnosed as tuberculous spondylitis and three cases that were initially diagnosed as pyogenic spondylitis. We reviewed the clinical, hematological, pathological and radiological characteristics.

Corresponding author: Jae Sung Ahn, MD

Department of Orthopedic Surgery, Chungnam National University College of Medicine

640 Daesa-dong, Joong-gu, Daejon, 301-721, Korea

Tel: +82-42-220-7349, Fax: +82-42-252-7098, E-mail: jsahn@cnu.ac.kr 
Table 1. Summary of cases

\begin{tabular}{ccccccccc}
\hline Case No. & Age/Sex & Affected site & Neurology & Fever & ESR & CRP & Initial impression & Dx. method \\
\hline 1 & 55/M & L2-3 & Intact & 36.5 & 64 & 9.2 & Tbc spondylitis & Medication change \\
2 & 48/M & L4-5 & L4 affected & 36.9 & 12 & 0.44 & Tbc spondylitis & Biopsy \\
3 & $57 / F$ & T11-L1 & Intact & 37.2 & 32 & 0.64 & Tbc spondylitis & Medication change \\
4 & 29/M & T11-L1 & Intact & 38.5 & 32 & 1.4 & Tbc spondylitis & Biopsy \\
5 & $67 / M$ & T11-T12 & Intact & 37.5 & 64 & 0.12 & Pyogenic spondylitis & Medication change \\
6 & 63/F & T12-L2 & Intact & 38.1 & 12 & 4.2 & Pyogenic spondylitis & Medication change \\
7 & 38/F & T12-L1 & Intact & 38.5 & 45 & 2.93 & Pyogenic spondylitis & Medication change \\
\hline
\end{tabular}

Tbc: tuberculous, ESR: erythrocyte sedimentation rate, CRP: C-reactive protein.
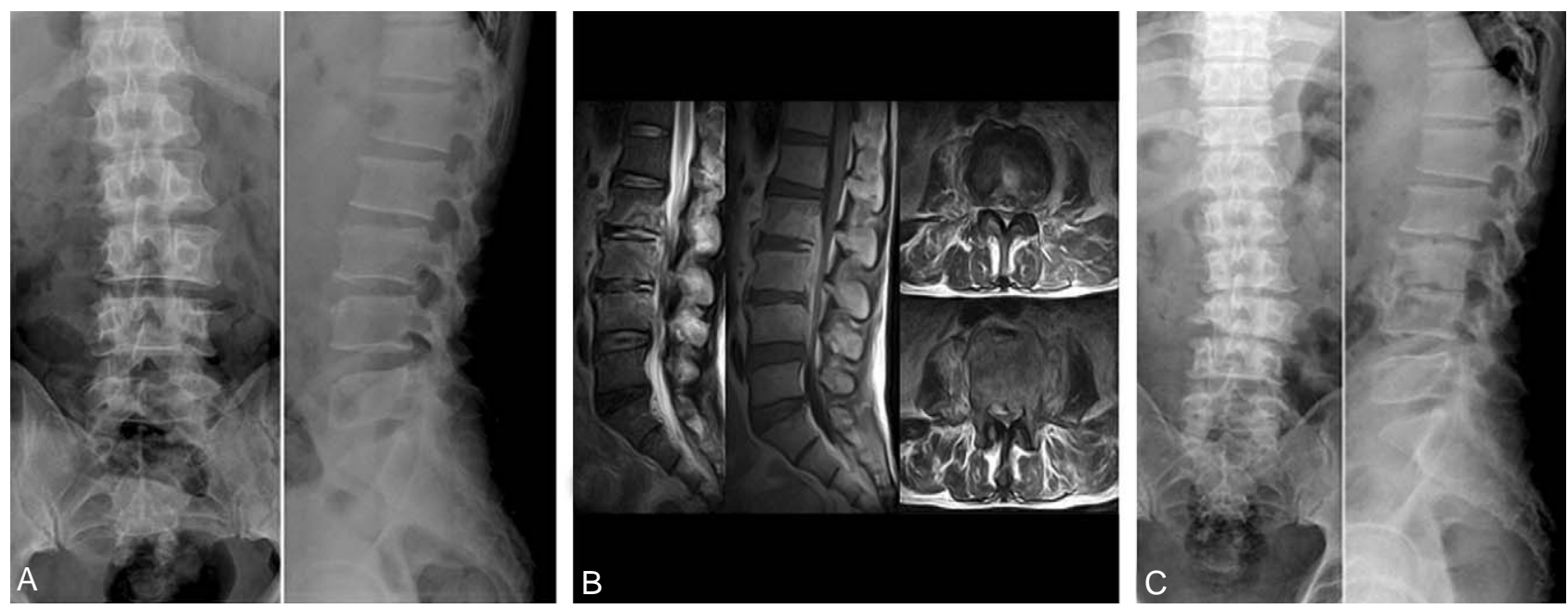

Fig. 1. Forty-eight years old male, initial simple X-rays shows mild scoliotic deformity and degenerative changes. (A) MRI shows vertebral height intact, paravertebral and epidural extension of the infectious process on L2, 3, 4 body with preserved adjacent intervertebral disc spaces. (B) Successful healing process is observed after HD \# 8 weeks.

\section{Materials and Methods}

Four cases that were initially diagnosed as pyogenic spondylitis but were finally confirmed to be tuberculous spondylitis were placed in Group 1, and 3 cases that were initially diagnosed as tuberculous spondylitis but confirmed as pyogenic spondylitis were placed in Group 2. We compared Group 1 and Group 2 patients for clinical manifestations, hematological findings, pathological findings and radiological findings. The average age of the patients was 51 years (range, 29 67 years); three patients were male and four patients were female. The most commonly affected site was the thoracolumbar spine ( 4 cases), followed by 2 the lumbar spine ( 2 cases) and the thoracic spine (1 case) (Table 1). We made a diagnosis based on clinical manifestations, a hematological examination including measurement of the erythrocyte sedimentation rate (ESR), level of C-reactive protein (CRP) and the use of radiological tests such as Xrays, a bone scan and MRI. Two cases were confirmed by a biopsy and the remaining five cases were confirmed by monitoring clinical symptoms and the laboratory tests while switching medication between anti-tubercular agents and other antibiotics. For Group 1, two patients were treated with resection of the affected site and posterolateral instrumentation and the other patients were treated with bed rest for more than 3 weeks, high alimentation, bracing and antitubercular medication. For Group 2, all patients were treated with bed rest for more than 4 weeks, high alimentation, bracing and the administration of intravenous antibiotics that were switched to oral medication after 2 months For Group 1 , all patients underwent anti-tubercular combination therapy containing four drugs for more than 12 months and were followed-up for 20 months. For Group 2, we prescribed second-generation cephalosporins with combined aminoglycoside and the patients were followed-up for 24 months.

\section{Results}

Four patients in Group 1 had fever $\left(37.4 \sim 38.5^{\circ} \mathrm{C}\right)$ at the 

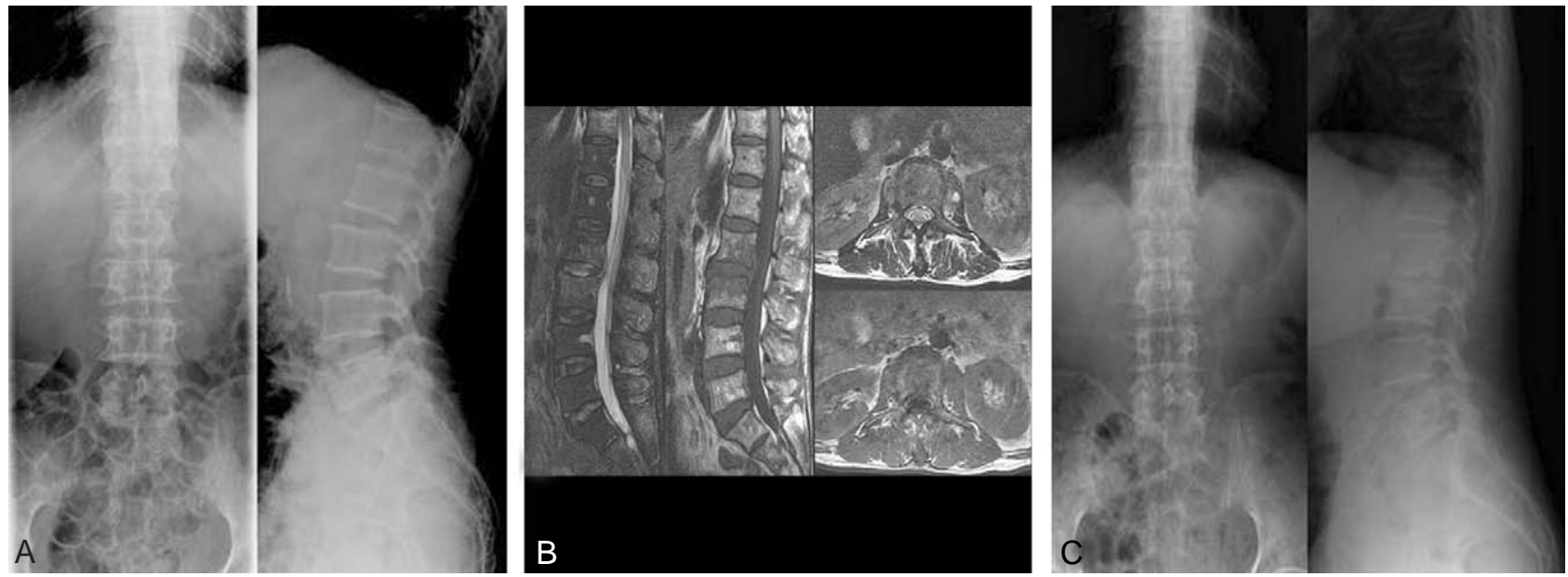

Fig. 2. 55 years old male, initial simple X-rays shows nonspecific findings except mild degenerative changes. (A) MRI shows mild compression of cecal sac at Lt. lateral space and minimal fluid signal intensity adjacent Lt. L3 with preserved adjacent intervertebral disc spaces. (B) Successful healing process is observed after HD \# 6 months.

time of hospitalization. Two patients had a normal ESR, white blood cell (WBC) count and CRP level. This group of patients was finally diagnosed with tuberculous spondylitis, as confirmed by an open biopsy and pathological study. Group 2 patients were treated with broad-spectrum antibiotics, but the symptoms aggravated. The symptoms improved with anti-tubercular therapy. Group 2 patients had no fever throughout the course of disease and the WBC count, ESR and CRP levels varied. The symptoms did not respond to anti-tubercular treatment but did respond to treatment with broad-spectrum antibiotics.

Bone union was observed in all cases in an average of 4 months (range, 3 6 months). The result of treatment differed based on the general condition of the patient. Two patients in Group 1 were treated by surgical resection and instrumentation. After surgery, the fever diminshed, local tenderness disappeared, hematological and radiological tests improved and the patients were able to walk with a thoracolumbosacral orthosis (TLSO) brace on the fifth postoperative day.

Fever that appeared in five cases diminished in 2 days (range, 1 4 days) after treatment with proper medication. Pain was reduced, blood tests improved and bony union was observed in radiographs (Figs. 1 and 2) after bed rest for 4 weeks and walking exercise in a TLSO brace was performed. In all cases, there were no recurrence and complications such as a neurological deficit or deformity and any metal failure in the operative cases.

\section{Discussion}


found in pyogenic spondylitis are that is that it does not involve the vertebrae, posterior arch and spinous process. Therefore, if the spondylitis involves the former mentioned structures, it is likely to be tuberculous spondylitis ${ }^{10,11}$. The ratio of pyogenic spondilitis between men and women is 1.5 3:1, which indicates that men are more easily affected. The early stage symptoms of pyogenic spondylitis are lower back pain, fever, loss of appetite, dullness, tenderness and stiffness. It shows a faster clinical course than tuberculous spondylitis. Symptoms of tuberculous spondylitis are back pain, partial tenderness and nighttime diaphoresis. In a hematological study, pyogenic spondylitis shows an increased or normal erythrocyte sedimentation rate, leukocyte count and CRP level. In tuberculous spondylitis, 80\% of the cases showed an increased erythrocyte sedimentation rate than an increase in the level of CRP. With plain X-rays, erosion of the column end plate can be seen, but sensitivity and specificity are very low. MR imaging has a reported sensitivity of $96 \%$, a specificity of $92 \%$, and an accuracy of $94 \%$ for the diagnosis of vertebral osteomyelitis ${ }^{12}$. Fatma et al..$^{13}$ mentioned that MRI evidence of disc space involvement was apparent in only $46 \%$ of lesions. A study of the signal intensity on T1- and T2-weighted images revealed a pattern that may be dissimilar to that commonly reported. Post-contrast enhancement adds more certainty to the diagnosis of tuberculous spondylitis. Liu et al. ${ }^{14}$ studied 29 cases of tuberculous spondylitis. Continuous two vertebral involvement, subligamental spread of paraspinal abscesses and cord indentation were observed in $93 \%$ of the cases. Destruction of the vertebral body occurred in $76 \%$ of the cases. These investrigators also concluded that Gd-DTPA administration did not facilitate diagnosis. Eugene ${ }^{15}$ performed a retrospective study on 103 cases of pyogenic spondylitis. In less than two weeks of symptoms, MRI appeared to give the correct diagnosis or suggest pyogenic vertebral osteomyelitis as a possible diagnosis in 55\% and $36 \%$ of cases, respectively. After two weeks, the percentage of correct and possible diagnoses of pyogenic vertebral osteomyelitis increased to $76 \%$ and $20 \%$, respectively. A diagnosis was made within one month in most cases. Martin et al. ${ }^{16}$ observed in a retrospective study on 122 cases of pyogenic and tuberculous spondylitis that the result of a biopsy provided a clear distinction between tuberculous and pyogenic spondylitis in $62.2 \%$ of cases, either by means of histology or by culture. Pain, gibbus formation and bony fusion gave no significant clues for the differential diagnosis. The combination of several unspecific findings such as patient history, erythrocyte sedimentation rate and radiographic assessment can lead to a correct diagnosis.

As in the previous studies, differentiation between pyogenic and tuberculous spondylitis has been made by clinical, hematological, radiological and pathological studies. In the present study of seven atypical cases, among the hematological, radiological and pathological evaluations, a pathological study is the most helpful. If the general condition of the patient is poor and the result of a biopsy is negative, starting treatment for the most likely diagnosis with periodic watching of the clinical, hematological and radiological changes is important for a precise diagnosis.

\section{Conclusions}

In infectious spondylitis, it is important to diagnose accurately based on the clinical presentations, laboratory findings, and radiological findings and by periodic evaluation of the response to treatment, which is important in atypical cases.

If there is no response or aggravation of symptoms despite treatment, the initial diagnosis must be re-evaluated, and the method of treatment must be changed according to the result.

\section{REFERENCES}

1. Kim NH, Lee HM, Suh JS: MRI diagnosis of tuberculous spondylitis. J Korean Orthop Assoc 1993; 28: 2512-2521.

2. Jeanneret B, Magerl F: Treatment of osteomyelitis of the spine using percutaneous suction/irrigation and percutaneous external spinal fixation. J Spinal Disord 1994; 7: 185-205.

3. Kim NH, Chung IH, Chung SM, Lee M: Pyogenic osteomyelitis of the spine. J Korean Orthop Assoc 1977; 12: 719-730.

4. Suk SI, Ahn JW, Cho HO: Clinical observation for pyogenic osteomyelitis of the spine. J Korean Orthop Assoc 1976; 11: 579-587.

5. Kim KS, Choi YS, Song JA, Kim DY: Posterior augumentation with rectangle luque for the treatment of tuberculosis of the lumbosacral junction in adults. J Korean Orthop Assoc 1996; 31: 292-301.

6. Safran O, Rand N, Kaplan L, Sagiv S, Floman Y: Sequential or simultaneous, same-day anterior decompres- 
sion and posterior stabilization in the management of vertebral osteomylitis of the lumber spine. Spine 1998; 23: 1885-1890.

7. Fu WK, Wu WC, Ip FK: Concomitent tuberculous and pyogenic infection of the cervical spine. Spine 1998; 23: 139-143.

8. Villoria MF, Fortea F, Moreno S, Munoz L, Manero M, Benito C: MR imaging and CT of central nervous system tuberculosis in the patient with AIDS. Radiol Clin North Am 1995; 33: 805-820.

9. Tekkok IH, Berker M, Ozcan OE, Ozgen T, Akalin E: Brucellosis of the spine. Neurosurgery 1993; 33: 838-844.

10. Babinchak TJ, Riley DK, Rotheram EB Jr: Pyogenic vertebral osteomylitis of posterior elements. Clin Infect Dis 1997; 25: 221-224.

11. Smith AS, Blaser SI: Infectious and inflammatory process of the spine. Radiol Clin North Am 1991; 29: 809-827.

12. Dagirmanjian A, Schils J, McHenry MC: MR Imaging of Spinal infections. Magn Reson Imaging Clin N Am 1999; 7: 525-538.

13. Al-Mulhim FA, Ibrahim EM, El-Hassan AY, Moharram HM: Magnetic resonance imaging of tuberculous spondylitis. Spine 1995; 20: 2287-2292.

14. Liu GC, Chou MS, Tsai TC, Lin SY, Shen YS: MR evaluation of tuberculous spondylitis. Acta Radiol 1993; 20: 554-558.

15. Carragee EJ: The clinical use of magnetic resonance imaging in pyogenic vertebral osteomyelitis. Spine 1997; 22: 780-785

16. Buchelt M, Lack W, Kutschera H, et al: Comparison of tuberculous and pyogenic spondylitis: an analysis of 122 cases. Clin Orthop Relat Res 1993; 296: 192-199. 